\title{
Labyrinthe
}

$13 \mid 2002$

Numéro 13

\section{Stéphane Beaud, ou la mise en actes de la recherche en sciences sociales}

Entretien avec Stéphane Beaud, maître de conférences en sociologie à l'université de Nantes et chercheur associé au laboratoire de sciences sociales de l'École normale supérieure, réalisé par Thierry Laurent, professeur stagiaire au lycée Henri-Wallon d'Aubervilliers, et Mathieu Rigo, du comité de rédaction de Labyrinthe.

\section{Stéphane Beaud}

\section{OpenEdition \\ Journals}

Édition électronique

URL : http://journals.openedition.org/labyrinthe/1485

DOI : $10.4000 /$ labyrinthe. 1485

ISSN : $1950-6031$

Éditeur

Hermann

Édition imprimée

Date de publication : 15 novembre 2002

Pagination : $9-29$

\section{Référence électronique}

Stéphane Beaud, "Stéphane Beaud, ou la mise en actes de la recherche en sciences sociales ", Labyrinthe [En ligne], 13 | 2002, mis en ligne le 23 février 2007, consulté le 19 avril 2019. URL : http:// journals.openedition.org/labyrinthe/1485; DOI : 10.4000/labyrinthe.1485

Ce document a été généré automatiquement le 19 avril 2019

Propriété intellectuelle 


\section{Stéphane Beaud, ou la mise en actes de la recherche en sciences sociales}

Entretien avec Stéphane Beaud, maître de conférences en sociologie à l'université de Nantes et chercheur associé au laboratoire de sciences sociales de l'École normale supérieure, réalisé par Thierry Laurent, professeur stagiaire au lycée Henri-Wallon d'Aubervilliers, et Mathieu Rigo, du comité de rédaction de Labyrinthe.

\section{Stéphane Beaud}

1 Son nouvel ouvrage, $80 \%$ au bac... et après ?', qui analyse sur près de dix ans les itinéraires des enfants de la démocratisation scolaire, est un appel au débat, comme l'était trois ans auparavant Retour sur la condition ouvrière ${ }^{2}$, écrit avec Michel Pialoux. C'est au lendemain du 21 avril que l'idée de cet entretien s'est imposée à nous. Nous avions noté l'accueil de $80 \%$, non seulement dans la presse mais aussi chez les enseignants, et après le choc du premier tour de l'élection présidentielle, nous avons cru nécessaire de reprendre certaines analyses du premier livre de Stéphane Beaud, Retour sur la condition ouvrière.

RINTHE - On vous a lu il y a peu dans Le Monde ${ }^{3}$, vu il y a quelques semaines dans l'émission Droits d'auteur de Frédéric Ferney sur France 5. Vivez-vous l'accueil de votre livre comme une forme de consécration, alors que le thème des $80 \%$ au bac est revenu au devant de l'actualité après sa publication?

STÉPHANE BEAUD - Consécration, c'est un bien grand mot... Il est difficile de se plaindre de voir son travail reconnu par les médias, et en même temps c'est une forme de reconnaissance qui est un peu angoissante. On peut se demander ce qu'elle signifie et surtout comment on sera compris car le sujet que j'ai traité, dans ce livre sur l'école, est très compliqué et, en plus, il est devenu très politique ${ }^{4}$. Ce livre, cela faisait longtemps que je l'avais en tête. Il y avait eu un article en 1997 dans la revue d'anthropologie Terrain, "Le temps élastique ${ }^{5}$ ", que j'ai repris et développé dans la seconde partie du livre - le passage sur l'aide à la révision des examens. Lors de l'enquête, j'ai eu conscience assez vite d'être tombé sur un sujet, non pas en or, mais très prometteur, en gestation. On voit affleurer, sur le terrain, une réalité sociale que l'on perçoit comme éminemment 
contradictoire, voire conflictuelle, et en même temps on s'aperçoit qu'elle n'est pas débattue. On sent des tiraillements: des professeurs qui ne sont pas contents de leur boulot, des élèves qui ne sont pas bien dans leur peau. Ce sont des situations d'entredeux, de malaise, qui sont très sociologiques et très intéressantes à analyser. En même temps, ce thème engage des questions politiques de grande importance: que fait-on de ces enfants de la massification scolaire, compte tenu de ce que le lycée professionnel est devenu ? Pour résumer, d'un côté, on est content de l'écho de son travail et, de l'autre, on est inquiet de la manière dont il va être utilisé. Ces jours-ci, Xavier Darcos revient sur ces $80 \%$ au bac, pas directement à la suite de ce que j'ai publié, je ne pense pas avoir une telle influence, mais c'est quelque chose qui était dans l'air. Quand j'ai écrit ce livre, j'avais aussi en tête les réactions syndicales, principalement les positions du Snes ${ }^{6}$, et ce qui me paraissait être un décalage entre leur position officielle (favorable aux $80 \%$ au bac) et une partie de leur base qui « grogne »...

4 LABYRINTHE - Votre dernier livre a rencontré plus d'écho que Retour sur la condition ouvrière. Pour le dire abruptement, cela ne pose-t-il pas le problème de la réception des sciences sociales par le champ politique, et celui d'une certaine méconnaissance politique des problèmes sociaux?

STÉPHANE BEAUD - C'est vrai. Quand Retour est sorti, il y a eu peu de chose dans la grande presse écrite (un entretien dans Libération). C'est le film Ressources humaines de Laurent Cantet qui, en janvier 2002, a relancé le livre. Cette réception en demi-teinte (par exemple, Le Monde n'en a fait un compte rendu que neuf mois plus tard et dans Le Monde de l'économie, pas dans Le Monde des livres) renvoyait, à mon avis, à des questions politiques: début 2000, la question ouvrière n'apparaissait pas du tout comme centrale, c'était même dans l'esprit de beaucoup de journalistes franchement archaïque. Et d'une certaine manière, à l'occasion de ce 21 avril funeste, qui a sauté à la figure de tout le monde, on s'aperçoit que c'est une partie de la théorie de Bourdieu qui est validée empiriquement. Tout à coup, l'ensemble du champ journalistique se saisit d'une question qu'il ne percevait pas du tout avant, et les journalistes qui cherchent à nous joindre sur le champ nous disent : « Vous avez raison, comment a-t-on fait pour ne pas voir? »

LABYRINTHE - Annie Collovald, sociologue elle aussi, vient de publier un texte d'humeur ${ }^{7}$ contre les commentateurs autorisés, qui posent dans un concert soudain l'équivalence entre vote $F N$ et vote ouvrier, ce qui est pour elle l'expression d'un racisme social latent dans les médias, qui va de pair avec le refus de considérer l'enracinement politique du FN. Acceptez-vous l'équivalence si stricte du vote ouvrier avec le vote Le Pen?

7 STÉPHANE BEAUD - Ce prêt-à-penser, c'est une question à soulever. C'est ce que l'on disait dans l'introduction de Retour: on pense aux ouvriers quand on veut percer l'énigme du vote Le Pen, quand on ferme des usines comme Moulinex... Le projet du livre et du travail avec Michel Pialoux, c'était d'abord de dire qu'il y a encore une "réalité ouvrière » à laquelle on peut s'intéresser, sans pour autant céder à la dictature du présent. Sur le vote ouvrier, c'est vrai que nous, nous ne travaillons pas par sondage. On a aussi fait très attention à ne pas nous entretenir avec des ouvriers sur le thème du lepénisme parce que l'on ne voulait surtout pas faire du "politisme ", en oubliant les effets de structure et de contexte. Il est difficile de solliciter quelqu'un sur ce thème, car encore aujourd'hui peut-être moins depuis deux mois - ce vote FN demeure peu connu, et même silencieux, voire honteux. Dans «Pourquoi la gauche a-t-elle perdu le vote populaire ? »" on insiste sur le côté honteux de ce vote qui bafoue des choses que l'on a apprises dans la famille, à l'école, même si l'on vient d'une famille ouvrière de droite : on ne se commet pas avec des gens comme Jean-Marie Le Pen dont le passé (jeux de mots antisémites, etc.) heurte les 
familles de résistants, ne passe pas. En revanche, en tant que sociologues, il y a un enjeu autour de la manière dont on travaille sur le vote Le Pen. Il ne faut pas se précipiter sur les explications toutes faites, comme le « vote de désespérance... » Il faut bien différencier les cas et, là, les sondages ne sont pas fiables. Il faut rappeler que le vote ouvrier aujourd'hui, c'est d'abord l'abstention. Les régions ouvrières déshéritées s'abstiennent, et par ailleurs on peut supposer qu'une partie du vote Le Pen chez les ouvriers serait plutôt le fait d'ouvriers qualifiés déclassés, de petits contremaîtres, de petits chefs, qui vivent très mal l'affaiblissement du groupe. Il y a une sorte d'homologie dans le déclassement entre les petits patrons et les ouvriers qualifiés, qui voient leur savoir-faire, leur professionnalisme et leur qualification contestés, qui se trouvent confrontés à un travail très ingrat, de chasse à l'absentéisme, etc. - qu'ils acceptent, parce qu'ils sont bien obligés de le faire.

Daniel Gaxie, dans Les Inrockuptibles, dit à juste titre qu'il ne faut pas se focaliser sur le vote des ouvriers non qualifiés, victimes du turnover et de la flexibilité : c'est plutôt chez ces catégories en déclassement que le ressentiment est énorme. Jacques Lévy, dans Libération', à partir de la cartographie, montre bien que le vote Le Pen est plus fort dans les zones géographiques où il y a ségrégation de l'espace, du pavillonnaire, etc., qu'en revanche dans les zones urbaines où il y a plus de brassage, de mixité sociale, il est plus faible.

9 Il y a donc cette piste des géographes. Il faut ensuite bien sûr sociologiquement en rendre compte, savoir ce que l'on peut faire dans ce domaine. Depuis dix ans, on assiste à un très fort mouvement de fuite, d'évasion des cités populaires, des « cités » comme l'on dit, et qui prend la forme d'un achat pavillonnaire à dix, vingt, et même cinquante kilomètres, avec pour conséquence la rupture des solidarités familiales - c'est à vérifier mais je pense que c'est assez juste, l'élévation du coût des transports, l'adoption d'un mode de vie plus onéreux (s'ajuster à une nouvelle norme de consommation de pavillonnaire), le repli sur soi, le face-à-face avec la télé..., et surtout la peur que les "problèmes des cités » débarquent en Seine-et-Marne, dans l'Eure, dans l'Oise...

LABYRINTHE - Quel est votre avis sur les zones rurales qui ont voté Le Pen alors qu'elles ignorent l'immigration, et dont on a beaucoup dit que leur vote avait été déterminé par la télévision?

11 STÉPHANE BEAUD - Là, c'est un peu différent. Il y a des zones rurales ouvrières. Dans les zones rurales traditionnelles, le pouvoir villageois a longtemps été aux mains des agriculteurs, qui tenaient le village. Je pense à un village dans l'Oise où, il y a vingt ans, on comptait trente-six agriculteurs; il n'y en a plus que six aujourd'hui. À l'époque, ils étaient tous RPR ; aujourd'hui, des ouvriers se sont installés en pavillonnaire, et le vote FN est devenu majoritaire : c'est une situation typique. Le vote Le Pen est vu comme une façon de se démarquer des "pauvres" qui menacent, pas forcément seulement les immigrés. Si l'on suit la chronique de Libération sur un petit village de Haute-Saône, on entend: "on » installe des immigrés, des jeunes étrangers, le deal, les «cassoces »comme on dit pour les cas sociaux -, les RMIstes; et là-dessus, pour redonner de l'expansion démographique aux petites communes, on a relogé des cas sociaux, la Caf a acheté des maisons qui sont louées pour 155 euros par mois à une famille de trois ou quatre enfants..., ces gens ne travaillent pas. Contre cela, il y a une réaction forte de la France laborieuse, qui travaille de plus en plus pour des salaires de smicards.

LABYRINTHE - Vous parliez, il y a déjà trois ans, de l'effet différencié des 35 heures selon les classes sociales, analyse qui a fait du chemin depuis le 21 avril. En tant que chercheur, n'avez-vous pas parfois l'impression de parler dans le désert? 
13 STÉPHANE BEAUD - À ce propos, une anecdote. En janvier 2001, Michel et moi, nous avons écrit, dans "Vive la sociale», une chronique de Libération, un article que nous avions intitulé "Les 35 heures vues d'en bas » et que Libération avait rebaptisé : «L'affront populaire». On commence par citer un ouvrier sur le thème «les 35 heures, grosse arnaque » : il balance en cinq minutes tout ce qui pour les ouvriers constitue le revers de la médaille des 35 heures, c'est-à-dire la sortie du temps de pause du temps de travail effectif (soit 36 heures 30 de travail au lieu de 35), la flexibilité, l'annualisation, le travail le samedi, les «horaires affichés » comme ils disent, plus les jours de vacances que l'on vous impose... Une semaine en hiver quand on n'a pas d'argent, ça n'intéresse pas les ouvriers. Et, surtout, la très forte rancœur à l'égard des cadres, qui prennent dix jours de congé absolument comme ils veulent. Donc, il y a chez les ouvriers le sentiment d'avoir été blousés dans l'affaire. C'est très intéressant en termes politiques : il y avait donc des remontées sur les effets négatifs des 35 heures en milieu ouvrier mais, par exemple, cet article n'a eu aucun écho. Par ailleurs, on sait que de nombreuses enquêtes sur les 35 heures avaient vraiment été orientées pour montrer que, finalement, cela marchait - ce que démentaient certaines réactions «sur le terrain" qui apparaissaient dans les enquêtes ethnographiques, etc. C'est d'ailleurs pour cela que je me dis que les financeurs de contrat dans les ministères devraient favoriser de bonnes enquêtes de terrain (on nous fait toujours le coup de la monographie, « pas représentative ») plutôt que d'envoyer des consultants qui font, vite fait, mal fait, des enquêtes dites représentatives, par sondage ou rapide campagne d'entretiens.

14 LABYRINTHE - En quoi, dans 80 \% et dans Retour, votre démarche se distingue-t-elle de ce que le pouvoir peut ainsi instrumentaliser, récupérer, voire prescrire?

15 STÉPHANE BEAUD - La question en elle-même est compliquée : comment « contrôler » les effets de ces recherches? Je différencierais les deux livres. Avec Retour, qui devait porter initialement un titre plus théorique («Les Ouvriers après la classe ouvrière »), on essayait de montrer qu'il n'y avait peut-être plus de classe ouvrière mais encore des ouvriers : le livre est un peu construit là-dessus. On peut dire qu'il a été fortement discuté. On a fait, grâce aux Amis du Monde diplomatique, des tournées dans toute la France - Lyon, Marseille, Besançon... Il y a eu de nombreux débats, du monde, des militants, des retraités, des petits fonctionnaires, des postiers... On voyait bien que ce livre correspondait à une attente : les débats dans les salles étaient vifs, parfois enflammés, les auditeurs refaisaient le monde et se livraient souvent à une autocritique, notamment sur l'impuissance des militants ouvriers à prendre en compte les problèmes des précaires et des chômeurs. Il y avait là comme une libération de parole : le livre existait, on pouvait en parler. Cela redonnait une valeur à la parole ouvrière, avec des réactions typiques comme : "On n'avait pas tort de penser ce qu'on pensait nous, même si à l'extérieur on disait que c'était pessimiste, archaïque, etc. » Là, pour Retour, il y a eu un effet de réhabilitation du politique, de ce mode critique que le monde ouvrier peut avoir.

En revanche, le point de départ des $80 \%$ au bac est différent: c'est un phénomène nouveau, une sorte de "révolution silencieuse " (comme dit Chauvel pour parler du creusement des écarts entre générations), qui voit croître le sentiment de ces titulaires de " petits bacs » d'avoir été blousés. Tout le problème est celui de l'après-coup : ce constat qu'ils font au terme de leur parcours n'est dicible que dans certaines conditions, face à moi qui les connais bien. Pendant très longtemps, et je suis sûr que pour vous c'est pareil, quand ils sont en route pour la terminale, la fac, ils y croient un peu ou ils font semblant d'y croire ; et puis, à un moment donné, ce n'est plus possible d'y croire, il leur faut faire 
le deuil de leurs aspirations scolaires. C'est à ce moment-là que les bouches se ferment. J'ai attendu un peu, que "mes » enquêtés soient plus mûrs, qu'ils réfléchissent sur ce qu'ils ont vécu pour qu'ils puissent commencer à dire ce qui pour eux est difficilement dicible. Bien sûr, j'étudie des cas individuels, ce qui n'est pas représentatif au sens statistique du terme (c'est une objection que l'on me fait de manière récurrente). En même temps, c'est la force et la vocation des « études de cas » que de pouvoir prétendre à un certain degré de généralisation. Ces cas individuels, je ne les ai pas choisis au hasard, on pourrait dire que je les ai construits chemin faisant, au cours de l'enquête.

17 LABYRINTHE - Si bien que le livre se lit comme une sorte de roman vrai plutôt qu'un essai sociologique «dur ». Vous percevez nettement votre travail comme politique, en militant...

STÉPHANE BEAUd - Pour dire les choses nettement, je conçois la sociologie comme Durkheim ou Weber la concevaient : une tradition critique. «La sociologie ne vaut pas une heure de peine si elle ne permet pas de tirer de l'ombre un problème social, et de lui donner un débouché politique. » Moi je fais de la sociologie pour dire que le monde social ne va pas de soi, et surtout ne va pas forcément bien; essayons de l'analyser, de nous donner des outils et de livrer des résultats aux politiques pour les aider à le voir autrement, et pas uniquement comme les experts le voient...

19 LABYRINTHE - Lors du colloque de Villepinte, en 1997, qui marque la conversion de la gauche majoritaire à une vision "décomplexée», comme l'on dit, de la sécurité, Lionel Jospin refuse que l'on accorde trop d'im-portance aux "excuses sociologiques" pour expliquer la délinquance. Ce sont sans doute ces « experts » qui ont soufflé à Lionel Jospin le thème des « excuses sociologiques ». Qu'en pensez-vous?

20 STÉPHANE BEAUD - Oui, c'est Jospin qui a utilisé cette expression. La logique du champ scientifique est parfois antinomique à celle du champ politique; et c'est en constatant une certaine impuissance politique face à des faits sociaux très "résistants" (les problèmes des cités et de la délinquance ne sont pas issus d'une génération spontanée mais sont le fruit d'une histoire sociale longue et multiforme) que Jospin s'est laissé aller à parler ainsi. Au-delà du cas Jospin et de son mode de formation politique (l'OCI), on voit quand même qu'il y a, à gauche, diverses traditions de la pensée qui acceptent très mal le regard sociologique, qui le trouvent - à tort - inutile ou démobilisateur. Si l'on prend une grande partie de la tradition trotskyste ou celle des militants ouvriers (du PCF stalinien, par exemple), dont la grille d'analyse est à l'époque très marxiste, le sociologue est perçu comme celui qui désespère Billancourt, qui coupe les cheveux en quatre, qui ergote. Si bien que l'on a vécu pendant des années sur la vérité établie de la classe ouvrière, avant-garde de l'histoire, etc. Il faudrait relire les critiques de Bourdieu par les marxistes dans les années soixante ${ }^{10}$; le weberianisme de Bourdieu-Passeron heurte alors le marxisme orthodoxe qui, dans la discipline sociologique, reste très abstrait et très peu fondé sur des enquêtes de terrain.

21 LABYRINTHE - Toujours sur le registre des "excuses sociologiques", la charpente de la vie sociale repose beaucoup, dans votre échantillon, sur le quartier, avec une incapacité presque pathétique des individus à s'affirmer dans ce cadre - ce que l'on voit dans la séquence où vous aidez le groupe à réviser ses examens -, comme si leur identité était adossée au quartier.

STÉPHANE BEAUD - Ce sont des cas choisis, il aurait fallu entrer dans l'analyse des petites différences qui font, au final, de grandes différences de parcours. Prenons les deux personnages principaux de la troisième partie du livre, Fehrat et Nassim : le premier a réussi il y a une dizaine de jours le concours d'éducateur, à l'âge de trente ans - cela 
faisait dix ans qu'il galérait dans les études; le second finit son contrat d'emploi-jeune et rêve d'entrer à la SNCF ou de prendre une conciergerie d'école. Or, Fehrat, parce qu'il est né dans un immeuble rénové, le "Berverly Hills » de Gercourt comme il dit avec humour, a bénéficié de ce "petit plus » résidentiel qui, au bout, fait nettement la différence : le « choix » des amis d'enfance, la scolarité dans une école primaire plus mixte socialement, une défiance quasi instinctive vis-à-vis de ceux qui habitent «Chicago " (nom donné au quartier de Nassim). C'est là que l'on mesure mieux la force contraignante du lieu de résidence, qui d'ailleurs s'exerce surtout sur ceux qui, de par toute leur histoire familiale, scolaire, etc., y sont en quelque sorte "assignés » et, de ce fait, ne peuvent pas s'approprier autre chose. Fehrat et Nassim habitent à quatre cents mètres l'un de l'autre, mais les blocs sont différents, les manières d'être sont différentes, les copains sont différents, alors qu'ils ont les mêmes parents, immigrés, OS, qu'il y a sept enfants d'un côté, dix de l'autre : ils vivent finalement des choses très différentes. On voit à quel point les petits capitaux de l'un ou ses contre-handicaps ont des effets à long terme très puissants. Parce que, si l'on creusait la comparaison, qu'est-ce qui fait que Nassim s'en sort avec difficulté ? C'est que, effectivement, il a un groupe, qu'il vit à travers ce groupe et que c'est comme ça qu'il a fabriqué son identité sociale. Tandis que Fehrat va être dans le groupe, mais il cherchera à s'en démarquer, parce qu'il ne veut pas être aspiré, happé.

C'est par ce désir, très inconscient chez lui, qu'il se donne la possibilité - je ne l'ai peutêtre pas assez dit - de s'en sortir. Il a compris assez tôt, intuitivement, que le quartier était un piège, et il a tenté, pour ainsi dire, dès le lycée, de s'en démarquer un peu : par exemple, il va s'ingénier à avoir au lycée des copains à l'extérieur du quartier, si possible des « Français ", aller dans les pavillons. Quand je l'ai vu la semaine dernière chez lui - il a un grand deux pièces à deux blocs de celui de son père à Gercourt, avec une grande pièce très peu meublée, un canapé, la télé et un grand bureau -, il m’a dit: «Tu vois, Stéphane, quand j'étais au lycée et que j'allais voir mes copains qui étaient pas ouvriers, à chaque fois c'est ça que je regardais, ils avaient un bureau. Et moi j'avais jamais eu de bureau de ma vie ; mon père ils nous a toujours fait la guerre quand on mettait l'électricité. Eh bien, la première chose que j'ai faite depuis que je suis autonome, c'est, regarde... », et il me montre le bureau, l'ordinateur, etc. Si l'on prend aussi bien Fehrat que Nassim (mais c'est le cas de la plupart de leurs copains du quartier), on sent bien qu'ils ont connu de vraies blessures sociales : ce sentiment d'être pauvres, de manquer de ce qui apparaît comme très ordinaire pour les autres enfants (une chambre à soi, un bureau, un tout petit peu d'argent de poche, de temps en temps partir en vacances...). Autre exemple : on a fait passer un long questionnaire en 1992 à 1200 élèves de troisième et on s'est aperçu que le taux de non-réponse à la question apparemment anodine « combien de frères et de sœurs avez-vous? » était le plus élevé. Parce qu'ils en avaient beaucoup, et qu'il est honteux d'appartenir à une famille nombreuse, d'une certaine manière, parce qu'on sait que ce n'est pas la norme.

LABYRINTHE - Cela ne marque-t-il pas une difficulté supplémentaire pour les enseignants?

STÉPHANE BEAUD - Lors de l'entretien avec Le Monde, j'aurais voulu développer davantage ce point, je l'ai fait de manière elliptique. Mon sentiment est qu'aujourd'hui il y a une distance sociale et culturelle qui va croissant entre les élèves d'une part, et les jeunes stagiaires et les néo-titulaires d'autre part. Et ce n'est pas sans malentendus et sans conséquences pédagogiques. On pourrait réduire cette distance en organisant le recrutement de bons étudiants issus de milieux populaires. Bien sûr, on peut être très bon professeur de banlieue en étant agrégé, normalien, mais pour accepter de faire ce boulot 
de professeur, il faut aussi pouvoir dépasser les a priori sur ces élèves. Ce qui me frappe le plus, c'est que, au moment où se profile un grand mouvement de renouvellement des enseignants, peu de monde se préoccupe de cette question, qui me paraît essentielle, de la vocation d'enseignant et, plus largement, de la manière de rallumer la flamme de la pédagogie dans les jeunes générations. À part la critique rituelle, et souvent exagérée, de l'IUFM.

Quand je vois Fehrat par exemple, qui est éducateur après des années de «pionnicat », et d'autres aussi, nombreux, qui n'ont même pas l'idée d'être enseignants parce qu'ils ont l'impression de viser trop haut, personne ne leur dit que c'est possible... C'est notamment à cause du concours de professeur des écoles qui est dorénavant à bac +3 , ce qui a entrainé une forte élévation du niveau du diplôme et un "embourgeoisement" du recrutement. Alors qu'il y a un tas de filles de banlieue, rencontrées dans mes enquêtes, qui sont très bien, qui ont un très bon rapport aux enfants mais qui se découragent (elles n'ont pas d'argent...), qui ne veulent pas attendre trois ans, qui passent des BTS, etc. C'est dommage, parce que je pense qu'elles auraient été de très bonnes institutrices, et auraient beaucoup donné pour aider les « gamins de cité » à s'en sortir.

Une partie de ces étudiants comprend intuitivement et sociologiquement ces jeunes. En même temps, ce n'est pas gagné, on peut être trop proche, ne pas savoir marquer ses distances... De toute façon, il faut de la mixité dans le recrutement des enseignants. Aujourd'hui, on perd cette ressource qui peut être utilisée, alors que l'on sait bien que pour se lancer en collège ou en lycée dans des zones difficiles, si l'on n'a pas la vocation, si l'on considère que son métier c'est d'abord enseigner, c'est-à-dire donner en nombre des devoirs, corriger en masse, on augmente évidemment les difficultés. L'expérience que j'ai de mes enquêtes et du lycée, où il y a beaucoup de jeunes professeurs qui arrivent, met le doigt sur la difficulté de s'adapter à son public: ce qu'il faut faire dans une certaine mesure, tout en restant ferme sur le niveau d'exigences; il y a un travail de conversion.

LABYRINTHE - Est-ce que cela aurait vraiment valeur d'exemple social ? Est-ce que ce discours de promotion sociale est simplement efficace dans la mesure où, certes, vous expliquez dans votre livre le découragement mais où, du point de vue des professeurs, cette vision républicaine, élitaire, peut laisser place au scepticisme?

STÉPHANE BEAUD - C'est monté dans le temps, une partie des classes a ce rejet-là. Pas toutes bien sûr! On pourrait distinguer les filières, mais aujourd'hui il y a une vraie hypocrisie ministérielle concernant les STT, les bacs professionnels. Même si une partie des élèves s'en sortira, il est évident qu'une masse restera sur le côté, et que la filière STT est trop importante en termes d'effectifs par rapport aux besoins de l'économie, aux débouchés. On voit bien qu'il y a des pôles de fixation dans les lycées, qu'il se dégage une humeur hostile aux professeurs, ce que Willis, dans le très beau texte «L'école des ouvriers $^{11}$ ", appelle une "culture antiécole» dans l'institution scolaire. On met des enfants d'ouvriers anglais sur les voies de garage de la formation professionnelle, et là, parce qu'ils savent qu'ils seront ouvriers, ils développent cette culture antiécole, ne veulent plus entendre parler des professeurs, qui sont des emmerdeurs, ni des bons élèves, qui sont des "fayots ». Cela ne va peut-être pas si loin en France, mais le phénomène s'est développé. Cette culture antiécole au lycée est un retournement, et une sacrée énigme pour les professeurs qui dispensent un savoir à des élèves qui, quasiment a priori, se mettent à le rejeter parce qu'ils sont en rupture avec l'institution scolaire. 
30 LABYRINTHE - De plus en plus d'élèves ont une activité salariée qui vient concurrencer leur activité scolaire, mais qui témoigne aussi d'une volonté de mobilisation, d'une certaine débrouillardise...

STÉPHANE BEAUD - C'est vrai que les caractéristiques, les qualités mobilisées ne sont pas $\mathrm{du}$ tout les mêmes, ce qui pose toujours la question de ces fameuses dispositions scolaires : comment vient ou ne vient pas ce rapport à l'école. J'imagine que vous voyez vous-mêmes dans vos classes en premier lieu la différence entre garçons et filles, qui était très marquée lors de mon enquête. Pour réussir scolairement, il faut des conditions matérielles mais aussi des dispositions : être relativement calme, tranquille, attentif en classe. Or, les lycéens en échec sont la plupart du temps «agités » : ils ne peuvent pas tenir en place une heure, une demi-heure. Il y a la sollicitation du quartier, la structuration psychologique, le fait qu'ils sont parfois d'une extrême tension intérieure : je dis souvent pour les définir que ce sont des "écorchés vifs", voulant toujours en découdre, se sentant en permanence victimes d'un complot du monde entier contre eux. Ces traits "psychologiques » sont antinomiques avec l'ascèse scolaire mais peuvent par ailleurs être des qualités - utilisées dans un certain type de travail salarié, par exemple chez McDonald's, où les "équipiers " disent volontiers que ce qu'ils aiment dans ce travail, c'est «bourrer", avec quasiment une dimension virile. Il faut aller vite et se défoncer, s'éprouver physiquement.

Quand je les aidais à réviser leur première année de fac, j'ai observé l'importance de cette question de la confiance en soi. Ils ne l'ont pas devant l'énoncé d'un devoir qui les amène de suite à penser : «Je n'y arriverai pas. » Ils ne le diront pas, parce qu'ils ont leur fierté, mais on voit très bien ce mouvement de recul, de défiance. C'est ce que le système scolaire, en laissant poursuivre des études à des élèves qui n'ont aucune confiance en eux, ne parvient pas à prendre en compte. Je ne dis pas que c'est spécifique aux « enfants de cité ", mais à ce niveau-là, le bât blesse, c'est ce que j'essayais de dire aussi dans l'entretien avec Le Monde: il faut rétablir, redonner un sens à la carrière scolaire, en montrant justement que l'on se construit dans les épreuves scolaires, que l'on franchit des paliers, alors que dans certains collèges, c'est le toboggan...

LABYRINTHE - On a insisté sur le thème du suivi individualisé, et l'institution incite à le pratiquer : quel est votre avis dans ce domaine?

STÉPHANE BEAUD - C'est une attente forte de la part des professeurs, qui renvoie à quelque chose qui n'est justement pas dans la tête du professeur qui a réussi le Capes ou l'agrégation. La demande affective de ces élèves peut les dérouter ou leur faire peur. Il faut à la fois l'accepter, savoir la " gérer » comme l'on dit aujourd'hui, et ne pas être trop proche... En fait, c'est très compliqué d'être professeur, cela demande un ensemble de qualités difficiles à réunir en une même personne...

LABYRINTHE - Sans compter que certains sous-entendus ou le langage gestuel - la tape sur l'épaule par exemple - ne sont pas du tout compris. L'institution des TPE ${ }^{12}$ et même la méthode de la mise en activité donnent souvent aux élèves le sentiment que ce qui est fait en classe, c'est un travail de moins à faire à la maison, ce que les professeurs finissent par accepter.

STÉPHANE BEAUD - Bernard Charlot, à Paris VIII, dit des choses assez intéressantes sur l'acquisition des savoirs, l'opposition des savoirs pratiques et théoriques. Sous prétexte de la résistance qui se fait jour aux savoirs scolaires, on a fait justement les ajustements dont vous parlez, avec des méthodes de pédagogie active, ou différenciée, qui ne sont pas inintéressantes, mais dont le risque est de faire passer à la trappe l'acquisition du savoir 
scolaire par le travail personnel. Dans les TPE, quel est le statut des élèves les plus largués? L'enjeu, aujourd'hui, c'est de ne pas oublier les bases, comme par exemple le fait d'apprendre, d'emmagasiner un minimum de connaissances. À la fac, je dis à mes élèves que les cours doivent s'apprendre : parfois ils n'en ont même pas l'idée, ils ne savent pas que l'histoire sociale (c'est le cours de première année que je fais) suppose une connaissance des processus, des dates, du contexte. Il y a aussi le problème de l'assiduité, mais ils peuvent se repasser les cours, sauf qu'au bout du compte il y a ceux qui ont simplement cessé d'apprendre. Sur le plan sociologique, l'interrogation porte sur le degré de négation, par l'IUFM et d'autres, de ce qui est spécifiquement le savoir des professeurs. Je trouve que le syndicalisme enseignant devrait davantage se poser ces questions, alors que c'est une des grandes interrogations des professeurs aujourd'hui.

LABYRINTHE - Dans les trajectoires sociales que vous étudiez, il y a une absence frappante de réflexion sur l'itinéraire, l'histoire des parents. Comment ces jeunes dont vous parlez ressentent-ils votre analyse, qui supplée les défauts de la leur?

STÉPHANE BEAUD - Ils s'étaient intéressés au livre, ils l'ont lu, et Fehrat a eu cette formule : «Tu nous as bien déshabillés. » Enfin, je ne faisais pas les entretiens forcément dans le but d'un livre; pendant très longtemps je ne leur ai pas dit, j'ai attendu pour sortir le livre. Fehrat est à peu près sorti d'affaire, Nassim est encore en difficulté et je le «secoue » un peu, comme un grand frère... Identité, ethnicité, c'est assez essentiel ; en fait, les jeunes que j'ai étudiés, c'est la génération qui vient après la marche des beurs. Ces derniers (Malek Boutih par exemple) ont été construits politiquement par ce mouvement social, ils ont cru à la "gauche » et se sont engagés, ils portaient en eux des dispositions au combat et à la révolte. Nassim et Fehrat, c'est la génération d'après, celle de la désillusion politique, scolaire, et aussi celle de l'instrumentalisation des beurs (dont le résultat est le passage à droite d'une Tokia Saïfi). Sans compter la victoire, souvent inaperçue, de Le Pen, qui a été d'avoir imposé ce thème de l'immigration, cette idée d'une altérité éternelle des enfants d'immigrés, de ceux qu'on s'est mis à appeler, dans les médias et à la moindre occasion, « musulmans ». C'est tout ça que des jeunes comme mes enquêtés ont pris en plein visage pendant des années. Il y a différentes réactions, comme celle de la radicalisation islamique ${ }^{13}$; mais la plupart des enfants d'immigrés des "quartiers" sont plutôt dans une démarche de reconstruction identitaire, comme Nassim, d'une façon fantasmatique, à la recherche d'une communauté imaginée, d'une identité de repli sur l'Algérie ou le Maroc, qu'ils ne connaissent pour ainsi dire pas. Faute de trouver une place « ici ", ils sont à la recherche d'un ailleurs rêvé où ils n'auront pas à faire face aux injonctions contradictoires qu'ils affrontent dans leur vie quotidienne. Sur ce thème, plus j'avance dans mes recherches, plus le travail d'Abdelmalek Sayad ${ }^{14}$ me parait essentiel, littéralement incontournable.

LABYRINTHE - Il y a en tout cas un effet de stigmatisation de ces enfants d'immigrés, on a l'impression que ces gens ont peur de faire le moindre pas.

STÉPHANE BEAUD - Et finalement ils se protègent en étant « entre eux »... Les garçons, entre eux! Il y a une dizaine de jours, on m'a demandé de venir dans un lycée pour présenter la sociologie à des élèves de première et de terminale ES. C'était très intéressant : il y avait dix-huit filles, d'origine immigrée, maghrébine, africaine... et pas un garçon. De même, j'ai accompagné un garçon dans une agence d'intérim, un gars qui en privé a de la ressource ; or, devant un «patron », entre guillemets, il était paralysé, il ne pouvait pas parler... J'ai dû prendre la parole à sa place. 
41 LABYRINTHE - Ce que vous rattachez à la honte sociale. Ce que le discours politique refuse de traiter, aussi.

STÉPHANE BEAUD - Mais il est difficile de politiser des choses comme celles-là. On dit toujours que le sociologue se demande comment politiser les questions sociales; par exemple, dans le cas des ouvriers, il y a cet effort à faire pour dépasser un sentiment de honte quant au langage, à l'écriture de tracts en faisant des fautes d'orthographe, à une expression orale difficile... C'est ce que l'on a essayé de dire un peu maladroitement dans l'article du Monde où l'on parlait d'un aspect peut-être positif du stalinisme, tel que le décrit Pudal ${ }^{15}$, qui était une machine non pas à intégrer mais à représenter... Là, il n'y a pratiquement plus de représentation ouvrière à l'Assemblée nationale, après les années Waldeck-Rochet, Marchais ; il reste Maxime Gremetz, Georges Hage, mais bon...

STÉPHANE BEAUD - C'est là qu'il faut vraiment faire des analyses localisées, sur le vote FN en Alsace, dans le Nord, dans le Midi..., quand on regarde ce qui se passe dans le Midi, où il semble bien y avoir quand même une vraie adhésion - anti-immigrés, fascisante, puissamment xénophobe, avec un rejet total des étrangers...

Cette idée souvent évoquée à propos du Midi qui voit coexister les pieds-noirs et les Maghrébins (dont pas mal sont surexploités comme travailleurs agricoles), et où la guerre d'Algérie n'a jamais été digérée, me semble profondément juste. C'est quelque chose qui plane au-dessus ou, plutôt, qui est la toile de fond invisible d'un tas d'interactions et de relations entre «Français » et « Arabes »... Je suis frappé dans les entretiens que j'ai faits avec ces jeunes nés de parents algériens de la méconnaissance qu'ils ont de la guerre d'Algérie. C'est pour cela que, au-delà des qualités intrinsèques, proprement documentaires, du film récent de Patrick Rotman ${ }^{16}$, je suis gêné par sa perspective unilatérale (la torture et la violence de la guerre), assez sensationnaliste. Ce qui est ici complètement occulté, c'est, d'une part, la violence sociale fruit de cent trente ans d'une colonisation brutale et, d'autre part, la complexité des rapports sociaux (il y avait notamment des militants anticolonisateurs chez les "Français », en Algérie comme en métropole, que je sache...). Si l'on regarde la réception de ce type de film chez ces jeunes d'origine algérienne (j'étais sur mon terrain à ce moment-là), ça se transforme en : « Les Français, vous êtes une bande de salauds. » D’un côté, ça construit l'image « Français, tous bourreaux ", "Algériens, tous victimes » et, par ailleurs, il n'y a aucune compréhension du fait qu'il y avait des colons, des petits blancs et des pieds-noirs, des exploiteurs et des exploités... Il y a là un enjeu formidable et un déficit d'explication des historiens de la réalité de ce passé, ramené à la seule question de la torture. Moi qui travaille à la revue Genèses, notamment avec Gérard Noiriel, et qui ai la chance de connaitre de jeunes chercheurs qui travaillent sur l'histoire coloniale, je suis affolé de voir le retard pris par l'historiographie française contemporaine sur ces questions-là, notamment parce qu'elle n'est pas assez ouverte sur les autres sciences sociales et sur l'étranger. Le renouvellement des problématiques se fait toujours attendre, tout comme la diffusion des savoirs historiques sur la colonisation dans les programmes de lycée.

LABYRINTHE - Vous posiez la question du rapport à l'Islam. On a l'impression, lorsque l'on fait cours, que l'on en sait parfois plus que certains élèves musulmans, qui ignorent la date de l'hégire, etc. 

du symbole, et en même temps je n'étais absolument pas dupe. Comme je le dis souvent, l'arbre Zidane, milliardaire du foot, fils exemplaire, ne peut pas cacher la forêt : à savoir, les nombreux jeunes pris au piège de la cité et qui se sentent de plus en plus rejetés. $\mathrm{Si}$ l'on prend l'exemple du football, on s'aperçoit qu'il y a de moins en moins de « jeunes des banlieues » dans les centres de formation. Tout simplement parce que c'est très difficile de devenir un sportif de haut niveau, cela exige un degré de maturité et de structuration psychologique que beaucoup d'entre eux n'ont pas. L'effet Zidane peut avoir un effet à long terme, à travers une espèce de banalisation de l'étrangeté ${ }^{17}$, mais le plus important reste la nature des obstacles à "l'intégration", notamment la discrimination à l'emploi qui est à la fois si forte et si déniée... Si, sur deux ans, on l'avait vue baisser de manière très soutenue, on aurait pu parler d'un effet réel, mais il ne faut pas raconter d'histoires, ni se rassurer à bon compte. Sur le moment, cela a été très halbwachsien, une mémoire collective s'est créée là ${ }^{18}$ : j'ai fait un tas d'entretiens quelque temps après où l'on me disait : « On était tous unis. »Cela a joué un mois, deux mois, six mois, mais cela n'a pas suffi, les phénomènes structurels ont repris le dessus : le logement social délabré, les cités ghettoïsées, les classes à $60 \%$ composées d'enfants d'immigrés... Aujourd'hui, la situation dans les milieux populaires est tendue. Il ne faut pas se dissimuler le fait que l'espèce de contre-racisme que l'on voit se développer chez certains jeunes est un des plus sûrs aliments du racisme populaire (réactif, pas théorisé et qui se nourrit des multiples formes de la concurrence sociale dans la vie quotidienne).

51 Le contre-racisme de ces jeunes, c'est à la fois une forme de retournement du stigmate (la fierté d'être arabe, de son pays d'origine, de ses parents qui est exaltée de manière proportionnellement inverse au mépris ressenti dans le regard de l'autre), et une logique de provocation qui s'enracine dans toute une histoire qui fait que beaucoup d'entre eux, notamment ceux qui n'ont pas les ressources scolaires pour se défendre par les mots et/ ou par une soif de revanche sociale, se vivent comme des "rabaissés ", " mis à l'écart " (ce sont des termes que l'on retrouve fréquemment dans les entretiens). D'où l'émergence, puis la consolidation dans les cités (il faudrait analyser finement le rôle du rap dans cette configuration) d'une "culture de la provocation", typique de certaines fractions de ces groupes de jeunes et qui est très mal vécue par les autres habitants. Si on laisse aller les choses, cette culture-là, qui est très proche d'une logique d'autodestruction, risque de s'étendre. C'est justement contre cela qu'il faudrait lutter, à travers un travail éducatif de fond, d'éducation et de prévention..., de l'anti-Sarkozy, quoi ! La réalité qui n'est jamais dite à TF1 et dans les JT de la peur, c'est que des gamins ne partent jamais en vacances (pour l'été, le maire RPR de Sochaux a fermé sa très belle piscine, fréquentée massivement par les enfants de cité qui ne peuvent pas partir... Une mesure terrifiante, scandaleuse...). Or, si vous arrivez à les faire partir une semaine en vacances, même s'ils foutent le bordel, s'ils vont à la mer ou faire du vélo sur la route Napoléon, ils sortent du quartier, ils s'éclatent, ils font du sport, ils sont relativement 
protégés du racisme, ils découvrent des gens bien, des choses... Au niveau du travail social, il y a une véritable abdication, dont on retrouve l'effet dans ces provocations.

C'est ce qui se passe: depuis quelques années, des gens qui ont des enfants en ont marre et ont quitté le quartier ; seuls restent les plus tourmentés, ce qui retombe directement sur le collège et les professeurs. Il y a des choses à faire. C'est ce que montrent les travaux d'Agnès Van Zanten sur les Zep ${ }^{19}$, qui souligne le rôle structurant de l'équipe pédagogique : pourquoi la première semaine ne fait-on pas des réunions avec les jeunes professeurs pour cadrer les difficultés et le travail commun? Cela donne de bons résultats et, après, l'année est sauvée. Sinon il y a quantité de jeunes professeurs qui se font ratatiner dès le début de l'année, et qui n'arriveront jamais à récupérer leurs classes. D'où la mise en cause des proviseurs : c'est aberrant qu'il n'y ait pas plus de professeurs qui deviennent proviseurs, que l'on ne puisse pas être proviseur trois ans puis redevenir professeur... L'existence du corps des proviseurs est une aberration, en comparaison avec d'autres pays, l'Allemagne par exemple. C'est dramatique comme le métier de professeur est fermé, sans perspective de mobilité.

\section{NOTES}

1. Stéphane Beaud, $80 \%$ au bac... et après? Les enfants de la démocratisation scolaire, Paris, La Découverte, 2002.

2. Retour sur la condition ouvrière, Paris, Fayard, 1999.

3. Voir ses entretiens dans Le Monde du 12 juin 2002, et avec Michel Pialoux le 2 juin. Voir aussi Le Monde des livres du 8 mars pour la recension de $80 \%$.

4. Lors d'une conférence de presse, le 6 juin 2002, le nouveau ministre délégué à

l'enseignement scolaire, Xavier Darcos, déclarait : «Depuis 1995, nous constatons que le niveau stagne, voire recule un petit peu. En gros, nous sommes à un palier de $60 \%$ et plus, ce qui nous éloigne de l'objectif de $80 \%$ [...] qui n'est pas un objectif réaliste à nos yeux. » 5. «Le temps élastique. Étudiants de " cité » et examens universitaires », Terrain. Carnets du patrimoine ethnologique, 29, septembre 1997.

6. Le principal syndicat enseignant du secondaire, cible des attaques de Claude Allègre, qui le considérait comme le gestionnaire du statu quo à l'Éducation nationale, du fait principalement de son influence sur le système des mutations.

7. « Le "vote Le Pen" : la faute au populaire?», Vacarme, 20, 2002, pp. 23-29.

8. À gauche!, Paris, La Découverte, juin 2002.

9. 20 juin 2002.

10. Voir Frédérique Matonti, « La Double Illusion. La Nouvelle Critique, une revue du PCF (1967-1980) », thèse de sciences politiques, Paris I, 1996. À paraître.

11. Paul Willis, Actes de la recherche en sciences sociales, 24 novembre 1978, pp. 50-61.

12. Les travaux personnels encadrés, généralisés à la rentrée dernière dans les lycées, associent plusieurs enseignants sur des thèmes transversaux; ils sont critiqués par les enseignants, car l'horaire de leur matière en est diminué. 
13. Voir Stéphane Beaud et Olivier Masclet, « Un passage à l'acte improbable ? Notes de recherche sur la trajectoire sociale de Zacarias Moussaoui », French Politics and Society, été 2002.

14. Voir La Double Absence. Des illusions de l'émigré aux souffrances de l'immigré, Paris, Seuil, 1999.

15. Bernard Pudal, Prendre parti. Pour une sociologie historique du PCF, Presses de la FNSP, 1989.

16. Diffusé sur France 3 les 4, 5 et 6 mars 2002. Il s'agit du documentaire, L'Ennemi intime, qui rassemble des témoignages sur la torture et l'armée française en Algérie.

17. Gérard Noiriel et moi, nous avons essayé de développer ce point dans un article de Vingtième Siècle (« L'immigration et le football », $2^{e}$ trimestre 1990).

18. Allusion au célèbre texte de Maurice Halbwachs, La Mémoire collective.

19. L'École de la périphérie, Paris, Puf, 2001. 